\title{
A Fixed-Dose Combination Of Gemigliptin And Rosuvastatin Exhibits Similar Pharmacokinetics, Pharmacodynamics, And Safety Compared To That Of A Loose Combination In Healthy Subjects
}

This article was published in the following Dove Press journal:

Drug Design, Development and Therapy

\author{
Eunwoo Kim (D)' \\ Kyoung Ryun Park (D) \\ In-Jin Jang (iD) \\ Kyung-Sang Yu' \\ SeungHwan Lee ${ }^{1,2}$ \\ 'Department of Clinical Pharmacology \\ and Therapeutics, Seoul National \\ University College of Medicine and \\ Hospital, Seoul, Republic of Korea; \\ ${ }^{2}$ Clinical Trials Center, Seoul National \\ University Hospital, Seoul, Republic of \\ Korea
}

\begin{abstract}
Purpose: Fixed-dose combination (FDC) of gemigliptin and rosuvastatin may improve medication compliance of patients with comorbid type 2 diabetes and dyslipidemia. Pharmacokinetics (PK), pharmacodynamics (PD), and safety of gemigliptin/rosuvastatin 50/20 mg FDC was compared with a loose combination of individual tablets in healthy subjects.
\end{abstract}

Patients and methods: A randomized, open-label, single-dose, two-period, two-sequence, two-treatment crossover study was conducted. Subjects received FDC or a loose combination of gemigliptin $(50 \mathrm{mg})$ and rosuvastatin $(20 \mathrm{mg})$ during each period, with a 14-day washout. Serial blood samples were collected up to $72 \mathrm{hrs}$ after dosing to measure plasma concentrations of gemigliptin, its active metabolite LC15-0636, and rosuvastatin for PK assessment, and DPP-4 activity for PD assessment. PK and PD parameters were calculated using a noncompartmental method. Safety profiles were evaluated throughout the study.

Results: Thirty-seven subjects completed the study. The concentration-time profiles of gemigliptin, LC15-0636, and rosuvastatin were similar between FDC and loose combination, respectively. For each of the three compounds, the geometric mean ratios $(90 \%$ confidence interval) of $\mathrm{FDC}$ to loose combination for $\mathrm{C}_{\max }$ and $\mathrm{AUC}_{\text {last }}$ fell within the bioequivalence range of $0.8-1.25$. Inhibition of DPP-4 activity-time profiles after administration of FDC and loose combination was overlapping, and $\mathrm{I}_{\max }$ and $\mathrm{AUEC}_{\text {last }}$ were similar. Both FDC and the loose combination were well tolerated.

Conclusion: PK, PD, and safety profiles of gemigliptin, its metabolite, and rosuvastatin were similar between FDC and loose combination. The FDC of gemigliptin (50 mg) and rosuvastatin $(20 \mathrm{mg}$ ) can be used as an alternative to a loose combination, which is expected to improve patient compliance.

Keywords: DPP-4 inhibitor, statin, type 2 diabetes, dyslipidemia, pharmacokinetics

\section{Introduction}

Patients with type 2 diabetes frequently suffer from dyslipidemia. According to a retrospective study, $77.2 \%$ of patients with type 2 diabetes had hyperlipidemia, which is the third highest disease comorbid with type 2 diabetes. ${ }^{1}$ In these patients, cardiovascular disease is the leading cause of death, and appropriate medication is needed to reduce the risk of cardiovascular disease. ${ }^{2,3}$ To reduce the economic burden and mortality of patients with type 2 diabetes and dyslipidemia, and to
Department of Clinical Pharmacology and Therapeutics, Seoul National University College of Medicine and Hospital, I0I

Daehak-ro, Jongno-gu, Seoul 03080,

Republic of Korea

Tel +82-2-2072-3520

Fax+82-2-742-9252

Email leejh4I3@snu.ac.kr 
improve patient compliance, there is a need for the development of fixed-dose combinations (FDC) for treatment of both diseases.

DPP-4 inhibitors can be used as alternatives to metformin for treatment of mild hyperglycemia (A1C $<7.5 \%$ ), and are used as add-on drugs in combination with metformin in patients with $\mathrm{A} 1 \mathrm{C} \geq 7.5 \%{ }^{4}$ Gemigliptin is a potent DPP-4 inhibitor and has been used as a treatment for type 2 diabetes since approval in 2012 by the Ministry of Food and Drug Safety (Republic of Korea). ${ }^{5,6}$ Gemigliptin is metabolized by cytochrome P450 (CYP), resulting in conversion to the metabolite LC15-0636, which has 2-fold higher DPP-4 inhibitory potency in vitro than the parent compound. ${ }^{7,8}$ Gemigliptin, like other DPP-4 inhibitors, ${ }^{4}$ is associated with a low risk of hypoglycemia and is neutral to body weight according to previous studies. ${ }^{9,10}$

For patients with dyslipidemia and type 2 diabetes requiring drug therapy to lower cholesterol, ${ }^{4}$ statins are recommended as a first-line drug therapy. ${ }^{4}$ Statins lower the risk of cardiovascular events and death in these patients. ${ }^{4}$ Rosuvastatin was effective in reducing low-density lipoprotein cholesterol (LDL-C) levels and was well tolerated in patients with dyslipidemia and type 2 diabetes. ${ }^{11-13}$ Rosuvastatin is excreted as its parent form in feces, with little hepatic metabolism. ${ }^{14}$

According to a previous study, pharmacokinetic properties of rosuvastatin are not altered by gemigliptin and vice versa. ${ }^{15}$ The maximum daily dose of gemigliptin approved by the Ministry of Food and Drug Safety (Republic of Korea) is 50 $\mathrm{mg}$ (Zemiglo ${ }^{\circledR}$, LG Chem. 2017). The maximum daily dose of rosuvastatin is $20 \mathrm{mg}$ in Asia $\left(\right.$ Crestor $^{\mathbb{R}}$, AstraZeneca. 2016. Republic of Korea) due to higher systemic exposure to rosuvastatin in Asian subjects compared to caucasian subjects. ${ }^{16,17}$ Both gemigliptin and rosuvastatin are administered once daily, regardless of diet. ${ }^{5,18}$ A new FDC of gemigliptin and rosuvastatin was developed and is expected to improve convenience of use and increase medication adherence in patients with type 2 diabetes and dyslipidemia.

The aim of this study was to investigate the pharmacokinetics (PK), pharmacodynamics (PD), and safety of gemigliptin $(50 \mathrm{mg})$ and rosuvastatin $(20 \mathrm{mg})$ when administered as an FDC compared to a loose combination in healthy subjects.

\section{Materials And Methods Study Design}

This study was a randomized, open-label, single-dose, twoperiod, two-treatment, two-sequence crossover clinical trial.
Subjects were randomly assigned to the two sequences at a ratio of 1:1. In each period, subjects were admitted to the hospital 1 day prior to the administration of the study drug and were fasted overnight. Subjects then received a loose combination of gemigliptin $50 \mathrm{mg}$ (LG Chem., batch number GEM15518C) and rosuvastatin $20 \mathrm{mg}$ (AstraZeneca, batch number 60019434) or an FDC of gemigliptin/rosuvastatin 50/20 mg (LG Chem., batch number 16GS81-1) with 200 $\mathrm{mL}$ of water in the first period. After a 14-day washout period, the subjects received an alternate treatment in the second period. Drinking water was prohibited for $1 \mathrm{hr}$ before dosing and 2 hrs after dosing. Food intake was prohibited for 4 hrs after dosing.

Blood samples for PK and PD assessment were collected pre-dose and at $0.5,1,1.5,2,3,4,5,6,8,12,24$, 48, $72 \mathrm{hrs}$ post-dose. Approximately $8 \mathrm{~mL}$ of blood for PK analysis and $3 \mathrm{~mL}$ of blood for PD analysis were taken at each blood sampling through a venous puncture or a saline-locked angiocatheter placed in the upper arm vein.

This study was conducted under the approval of the institutional review board of Seoul National University Hospital (SNUH), and the Korea Ministry of Food and Drug Safety (ClinicalTrials.gov registry number: NCT02670070). The study was conducted in accordance with the ethical guidelines of the Declaration of Helsinki and Good Clinical Practice of International Conference on Harmonization. Written informed consent was obtained from all subjects before conducting the screening test for participation in this study.

\section{Subjects And Treatments}

Healthy male subjects aged 19-45 years with a body mass index of $18-27 \mathrm{~kg} / \mathrm{m}^{2}$ and fasting glucose of $70-125 \mathrm{mg} / \mathrm{dL}$ at screening were enrolled in this study. Subjects who had clinically significant abnormalities during physical examination, electrocardiogram monitoring test, clinical laboratory tests, or vital signs at screening were excluded. In addition, subjects who had hypersensitivity reactions to gemigliptin or rosuvastatin and who had a history of drug-induced muscle disease were excluded.

Sample size was calculated based on prior study results, from which the intra-subject coefficient of variation of the major pharmacokinetic parameters was assumed to be about $20 \%$ for gemigliptin and about $30 \%$ for rosuvastatin. Assuming a dropout rate of $20 \%$, inclusion of 40 subjects was estimated to result in $80 \%$ power at a significance level of 0.05 to detect whether the $90 \%$ confidence interval $(90 \% \mathrm{CI})$ for comparison of 
pharmacokinetic parameters of FDC to loose combination was within the bioequivalence range of $0.8-1.25 .{ }^{19}$

\section{Determination Of Plasma Drug Concentration And DPP-4 Activity}

Blood samples for PK assessment were collected in heparinized tubes and centrifuged at $1,800 \mathrm{rpm}$ for 8 mins at $4^{\circ} \mathrm{C}$. After centrifugation, plasma samples for gemigliptin analysis were transferred to tubes containing an equal amount of $5 \%$ formic acid solution for long-term stability of gemigliptin. ${ }^{8}$ Plasma samples for rosuvastatin analysis were placed in tubes after centrifugation without any additional treatment. Blood samples for PD assessment were collected in EDTA tubes and centrifuged. All plasma samples for PK and PD assessment were placed in a $-20{ }^{\circ} \mathrm{C}$ freezer immediately after pretreatment (if necessary) and stored at $-70^{\circ} \mathrm{C}$ within $24 \mathrm{hrs}$ until sample analysis.

Plasma concentrations of gemigliptin, LC15-0636, and rosuvastatin were analyzed using liquid chromatography tandem mass spectrometry (LC-MS/MS; Shiseido NASCA and AB Sciex Triple Quad 5500). Gemigliptin, LC150636, and rosuvastatin were separated using a UK-C18 column ( $3 \mu \mathrm{m}, 50 \times 2 \mathrm{~mm}$, Imatackt, Japan), and detected by positive electrospray ionization with multiple reaction monitoring (AB Sciex Triple Quad 5500).

The analytical method was validated across the range of $0.5-200 \mathrm{ng} / \mathrm{mL}$ for gemigliptin, and $0.25-100 \mathrm{ng} / \mathrm{mL}$ for LC15-0636. For gemigliptin, the mean accuracies of the quality control (QC) samples were within 103-105\% and the coefficient of variation $(\mathrm{CV})$ was $\leq 4.46 \%$. For LC15-0636, the corresponding values were $102-104 \%$ and $\leq 6.33 \%$, respectively. The analytical method was validated across the range of $0.25-100 \mathrm{ng} / \mathrm{mL}$ for rosuvastatin, and the mean accuracies of the quality control samples were within $90.7-101 \%$ and the $\mathrm{CV}$ was $\leq 5.88 \%$. All samples of FDC and loose combination were analyzed according to the validated method as described above.

Plasma DPP-4 activity was determined using a continuous spectrophotometric assay as described in a previous study. ${ }^{20}$

\section{Pharmacokinetic/pharmacodynamic Assessment}

Pharmacokinetic (PK) and pharmacodynamic (PD) assessments were performed on subjects who completed all planned PK and PD blood sampling. Calculation of PK and PD parameters was performed using Phoenix ${ }^{\circledR}$
WinNonlin ${ }^{\circledR}$ software, version 7.0 (Certara USA Inc., Princeton, NJ, USA) using the non-compartment method. For PK assessment, area under the concentration-time curve from 0 to the last measurable concentration $\left(\mathrm{AUC}_{\text {last }}\right)$ and maximum concentration $\left(\mathrm{C}_{\max }\right)$ were calculated. For PD assessment, the area under the inhibition of DPP activity-time curve from 0 to the last measurable concentration $\left(\mathrm{AUEC}_{\text {last }}\right.$ ) and maximum inhibition of DPP-4 activity $\left(\mathrm{I}_{\max }\right.$ ) were calculated using change from the basal value. All statistical analyses were performed using SAS version 9.4 (SAS Institute Inc., Cary, NC, USA). The PK and PD parameters were log-transformed and the geometric mean ratio (GMR) of FDC to loose combination and its $90 \%$ CIs were estimated using a linear mixed-effect model. The model contained period, sequence, and treatment as fixed effects, and subject nested within sequence as a random effect. It was concluded that there were no significant PK differences between the two treatments when GMR $(90 \% \mathrm{CI})$ of FDC to loose combination was contained within the bioequivalence range of $0.8-1.25$.

\section{Safety Assessment}

Safety evaluation was performed on subjects who received the study drugs at least once. All subjects were examined for adverse events (AEs), physical examination, electrocardiogram test, clinical laboratory tests, and vital signs. The results of each assessment were reviewed to determine their clinical significance and to determine whether they were related to study drugs.

\section{Results Study Population}

A total of 42 subjects were enrolled in the study, two of whom were withdrawn due to abnormal ECG test results before the first administration. Forty subjects received the study drug at least once and these subjects were evaluated for demographic characteristics and safety. After the first administration and before the second administration, two subjects were withdrawn due to withdrawal of consent and use of concomitant medication without investigator approval. Another subject was dropped due to an adverse event (gastroenteritis) which was unlikely related to study drug. A total of 37 subjects completed the study and these subjects were evaluated for PK and PD. Demographic characteristics are summarized in Table 1. There were no 
Table I Demographic Characteristics Of The Subjects

\begin{tabular}{|l|l|l|l|}
\hline & Sequence A $(\mathbf{N}=20)$ & Sequence B (N = 20) & p-value \\
\hline Age (years) & $28.5 \pm 5.3(21-42)$ & $28.1 \pm 5.6(21-44)$ & $0.6640^{*}$ \\
Height $(\mathrm{cm})$ & $176.4 \pm 7.0(165-186)$ & $173.5 \pm 5.3(161-180)$ & $0.1753^{*}$ \\
Weight $(\mathrm{kg})$ & $72.3 \pm 9.2(56.2-87.2)$ & $68.8 \pm 7.3(53.0-85.6)$ & $0.1996^{* *}$ \\
BMI $\left(\mathrm{kg} / \mathrm{m}^{2}\right)$ & $23.2 \pm 2.3(18.5-26.9)$ & $22.9 \pm 2.1(18.3-27.0)$ & $0.6103^{* *}$ \\
\hline
\end{tabular}

Notes: Data are presented as arithmetic mean \pm standard deviation. (min-max). Sequence A was first administered the loose combination of gemigliptin (50 mg) and rosuvastatin $(20 \mathrm{mg})$, followed by the fixed-dose combination of gemigliptin/rosuvastatin $50 / 20 \mathrm{mg}$, and sequence B was administered in the reverse order. * P-value was obtained from Mann-Whitney U-test. ** P-value was obtained from two-sample $t$-test.

significant differences in subject characteristics between the two sequences (Table 1).

\section{Pharmacokinetic/pharmacodynamic Results}

Plasma concentration-time profiles and PK parameters of gemigliptin, LC15-0636, and rosuvastatin after administration of FDC and loose combination were superimposed (Figure 1). The GMR (90\% CI) of gemigliptin $\mathrm{C}_{\max }$ and $\mathrm{AUC}_{\text {last }}$ for FDC to loose combination was $1.08(0.99-1.18)$ and 1.02 (0.96-1.09), respectively. The GMR (90\% CI) of rosuvastatin $\mathrm{C}_{\max }$ and $\mathrm{AUC}_{\text {last }}$ for $\mathrm{FDC}$ to loose combination was 1.09 (0.96-1.22) and 1.02 (0.95-1.09), respectively. In addition, the corresponding values of LC15-0636 were $1.00(0.93-1.08)$ and 1.02 (0.97-1.08). Overall, the GMR $(90 \% \mathrm{CI})$ of the PK parameters of gemigliptin and rosuvastatin fell within the bioequivalence criteria of $0.8-$ 1.25, which shows that systemic exposure is similar between the two treatments (Table 2).

DPP-4 activity inhibition-time profiles after administration of FDC and loose combination were superimposed (Figure 2). Inhibition of plasma DPP-4 activity following FDC and loose combination administration was similar when evaluated by $\mathrm{AUEC}_{\text {last }}$ and $\mathrm{I}_{\max }$. The GMR (90\% CI) of $\mathrm{AUEC}_{\text {last }}$ and $\mathrm{I}_{\max }$ for FDC to loose combination was 1.00 (0.98-1.03) and 1.01 (1.00-1.02), respectively (Table 3).

\section{Safety Results}

The incidence of drug-related AEs was similar between loose combination and FDC. A total of 2 drug-related AEs (neck pain and upper respiratory tract infection) occurred in 2 subjects after administration of loose combination, and 3 drug-related AEs ( 2 cases of oropharyngeal pain and 1 case of cough) occurred in 3 subjects after administration of FDC. The subject who had upper respiratory tract infection was administered xylometazoline $\mathrm{HCl}$ (Metarivin $0.1 \%$ ) without investigator approval and was removed from the study because of the possibility of interaction between xylometazoline and the study drugs. All drug-related AEs were mild and there was no serious AE. In addition, all patients who experienced AEs successfully recovered and remained in the study. There were no clinically significant changes in physical examinations, electrocardiograms, clinical laboratory tests, or vital signs.

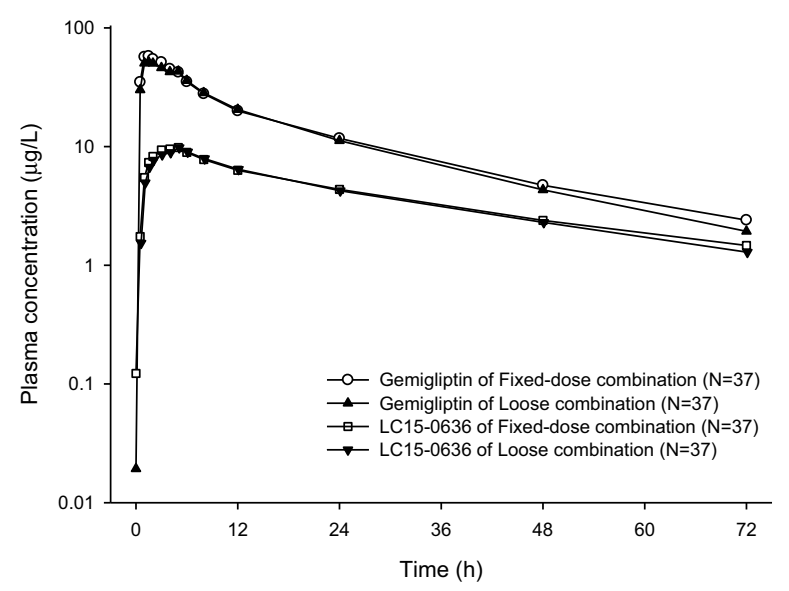

(a)

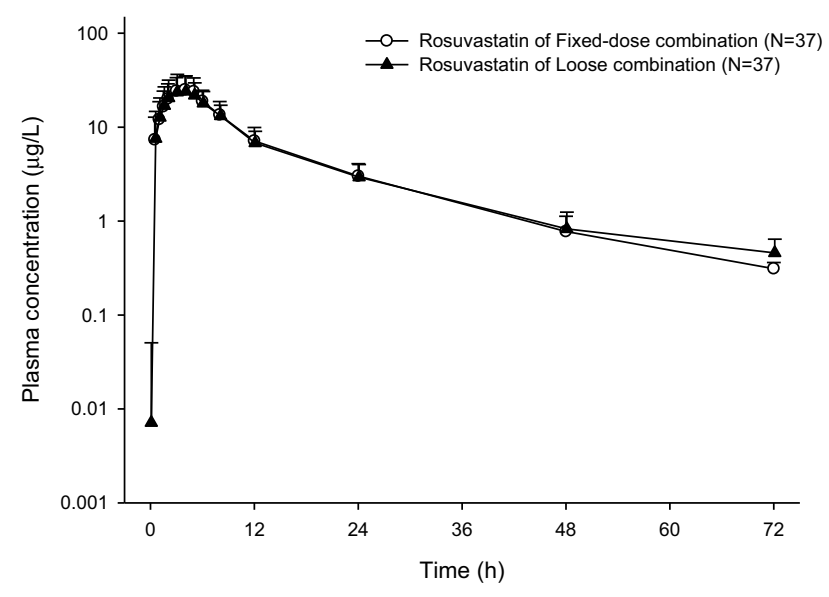

(b)

Figure I Mean plasma concentration-time profiles of (A) gemigliptin and LCI50636 and (B) rosuvastatin after a single administration of a fixed-dose combination and loose combination of gemigliptin $50 \mathrm{mg}$ and rosuvastatin $20 \mathrm{mg}$. Error bars represent standard deviations. 


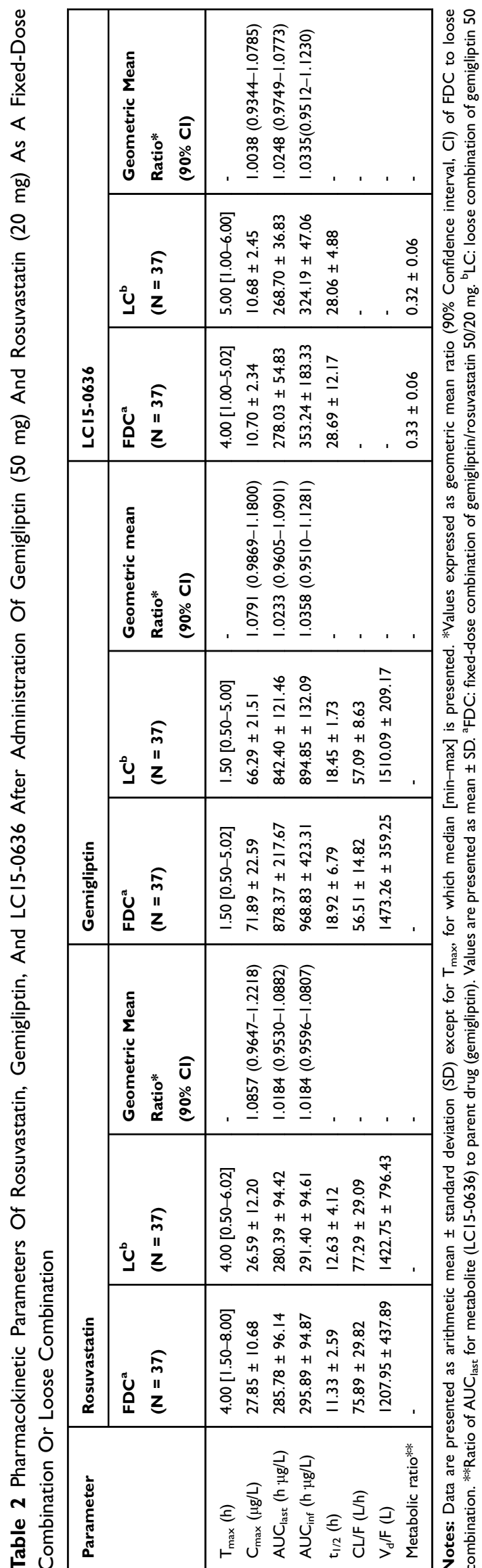

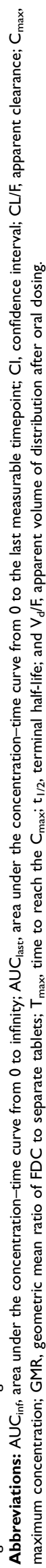

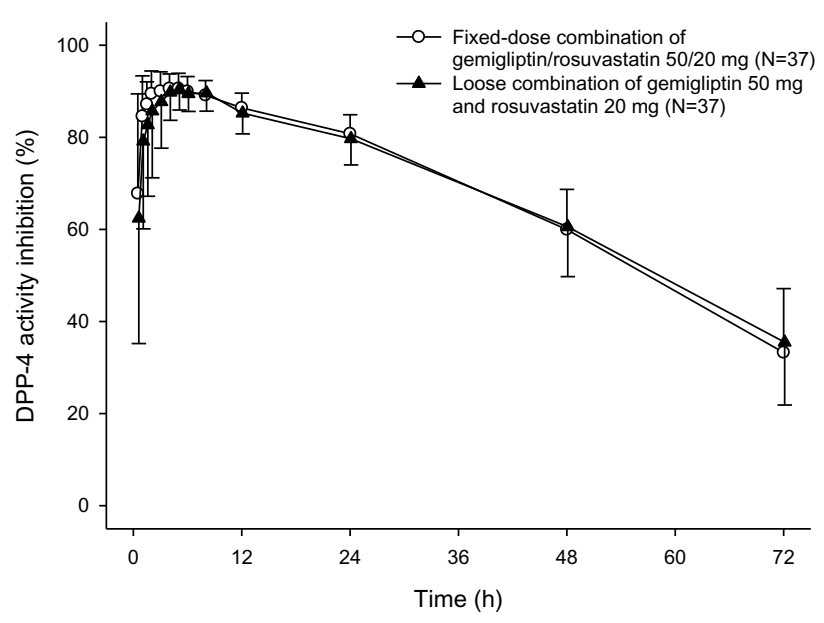

Figure 2 Mean plasma DPP-4 activity inhibition from baseline-time profiles after administration of a fixed-dose combination and loose combination of gemigliptin and rosuvastatin. Error bars represent standard deviations.

\section{Discussion}

This study was conducted to evaluate PK, PD, and safety of FDC of gemigliptin $(50 \mathrm{mg})$ and rosuvastatin $(20 \mathrm{mg})$ compared to loose combination. The GMR and $90 \% \mathrm{CI}$ of $\mathrm{C}_{\max }$ and $\mathrm{AUC}_{\text {last }}$ for gemigliptin, its metabolite LC150636, and rosuvastatin fell within the bioequivalence criteria of $0.8-1.25$. The GMR and $90 \%$ CI of AUEC and $\mathrm{I}_{\max }$ for DPP-4 were also within the range of $0.8-1.25$. In addition, all AEs were mild, and the number of AEs and the number of subjects who developed AEs were similar for both formulations. Overall, the two formulations were found to be similar in PK, PD, and safety aspects. This suggests that FDC may be a new, convenient option for patients with type 2 diabetes and dyslipidemia.

The washout period of this study was 14 days, which is long enough to eliminate carryover effects considering previously reported half-lives of $17 \mathrm{~h}$ for gemigliptin, $27 \mathrm{~h}$ for LC15-0636, and $19 \mathrm{~h}$ for rosuvastatin. ${ }^{9,15,21}$ However, one case of carryover effect for gemigliptin and rosuvastatin was observed at pre-dose in the second period after washout. However, PK analysis was performed without any adjustments because both of gemigliptin and rosuvastatin concentrations were less than $5 \%$ of $\mathrm{C}_{\max } \cdot{ }^{19}$

In this study, DPP-4 inhibition by gemigliptin was evaluated as a PD parameter. DPP-4 inhibition increases glucagon-like peptide 1 (GLP-1) and glucose-dependent insulinotropic polypeptide (GIP) levels, thereby increasing insulin secretion and ultimately lowering glucose levels. ${ }^{22}$ However, PD parameters of rosuvastatin were not evaluated in this study because changes in cholesterol synthesis induced by rosuvastatin are not immediate as LDL-C is 
Table 3 Comparison Of Plasma DPP-4 Activity Inhibition From Baseline After Administration Of Gemigliptin (50 mg) And Rosuvastatin $(20 \mathrm{mg})$ As A Fixed-Dose Combination Or Loose Combination

\begin{tabular}{|c|c|c|c|}
\hline \multirow[t]{2}{*}{ Parameter } & \multicolumn{3}{|c|}{ DPP-4 Activity Inhibition (\%) } \\
\hline & $\begin{array}{l}\text { Fixed-Dose Combination } \\
(\mathbf{N}=37)\end{array}$ & $\begin{array}{l}\text { Loose Combination } \\
(\mathrm{N}=37)\end{array}$ & $\begin{array}{l}\text { Geometric Mean Ratio* } \\
(90 \% \mathrm{Cl})\end{array}$ \\
\hline $\operatorname{AUEC}_{\text {last }}(\% \cdot h)$ & $4838.76 \pm 442.36$ & $4826.99 \pm 511.14$ & I.004I (0.9797-I.029I) \\
\hline$I_{\max }(\%)$ & $93.35 \pm 2.58$ & $92.65 \pm 3.78$ & $1.0082(0.9956-1.0210)$ \\
\hline
\end{tabular}

Note: *Values expressed as geometric mean ratio $(90 \% \mathrm{Cl})$ of fixed-dose combination to loose combination. Data are presented as arithmetic mean \pm standard deviation. Abbreviations: $\mathrm{AUEC}_{\text {last }}$, area under the inhibition of DPP activity-time curve from 0 to the last measurable timepoint; $\mathrm{Cl}$, confidence interval; $I_{\text {max }}$, maximum inhibition of DPP-4 activity.

reduced by $64 \%$ in the first week after rosuvastatin administration and by $90 \%$ in the second week. ${ }^{23}$

\section{Conclusion}

$\mathrm{PK}, \mathrm{PD}$, and safety profiles of gemigliptin, its metabolite, and rosuvastatin were similar between FDC and loose combination. The FDC of gemigliptin $(50 \mathrm{mg})$ and rosuvastatin $(20 \mathrm{mg})$ can be used as an alternative to loose combination, which is expected to improve patient compliance.

\section{Data Sharing Statement}

The authors do not intend to share substantial data of this study, but they are ready to share de-identified file of substantial data in excel format and all other study-related documents, at any specific time for any period, if the editorial board requires.

\section{Acknowledgment}

This study was funded by LG Chem., Seoul, Republic of Korea.

\section{Disclosure}

Kyoung Ryun Park is currently employed by Boryung Co., Ltd. Her contribution to the manuscript was based on her prior employment, and the current manuscript does not reflect any position of Boryung Co., Ltd. The authors report no other conflicts of interest in this work.

\section{References}

1. Iglay K, Hannachi H, Joseph Howie P, et al. Prevalence and coprevalence of comorbidities among patients with type 2 diabetes mellitus. Curr Med Res Opin. 2016;32(7):1243-1252. doi:10.1185/ 03007995.2016.1168291

2. Khavandi M, Duarte F, Ginsberg HN, Reyes-Soffer G. Treatment of dyslipidemias to prevent cardiovascular disease in patients with type 2 diabetes. Curr Cardiol Rep. 2017;19(1):7. doi:10.1007/s11886-0170818-1
3. Mooradian AD. Dyslipidemia in type 2 diabetes mellitus. Nat Clin Pract Endocrinol Metab. 2009;5(3):150-159. doi:10.1038/ncpendmet1066

4. Garber AJ, Abrahamson MJ, Barzilay JI, et al. Consensus statement by the American Association of Clinical Endocrinologists and American College of Endocrinology on the comprehensive type 2 diabetes management algorithm - 2018 executive summary. Endocr Pract. 2018;24(1):91-120. doi:10.4158/CS-2017-0153

5. Kim SH, Yoo JH, Lee WJ, Park CY. Gemigliptin: an update of its clinical use in the management of type 2 diabetes mellitus. Diabetes Metab J. 2016;40(5):339-353. doi:10.4093/dmj.2016.40.5.339

6. Kim SH, Lee SH, Yim HJ. Gemigliptin, a novel dipeptidyl peptidase 4 inhibitor: first new anti-diabetic drug in the history of Korean pharmaceutical industry. Arch Pharm Res. 2013;36(10):1185-1188. doi:10.1007/s12272-013-0171-x

7. Noh YH, Lim HS, Jin SJ, et al. Effects of ketoconazole and rifampicin on the pharmacokinetics of gemigliptin, a dipeptidyl peptidase-IV inhibitor: a crossover drug-drug interaction study in healthy male Korean volunteers. Clin Ther. 2012;34(5):1182-1194. doi:10.1016/j. clinthera.2012.04.001

8. Kim N, Patrick L, Mair S, et al. Absorption, metabolism and excretion of [14C]gemigliptin, a novel dipeptidyl peptidase 4 inhibitor, in humans. Xenobiotica. 2014;44(6):522-530. doi:10.3109/00498254.2013.865856

9. Lim KS, Kim JR, Choi YJ, et al. Pharmacokinetics, pharmacodynamics, and tolerability of the dipeptidyl peptidase IV inhibitor LC15-0444 in healthy Korean men: a dose-block-randomized, double-blind, placebocontrolled, ascending single-dose, phase I study. Clin Ther. 2008;30 (10): 1817-1830. doi:10.1016/j.clinthera.2008.10.013

10. Yang SJ, Min KW, Gupta SK, et al. A multicentre, multinational, randomized, placebo-controlled, double-blind, phase 3 trial to evaluate the efficacy and safety of gemigliptin (LC15-0444) in patients with type 2 diabetes. Diabetes Obes Metab. 2013;15(5):410-416. doi:10.1111/dom.12042

11. Tuomilehto J, Leiter LA, Kallend D, A review of the efficacy of rosuvastatin in patients with type 2 diabetes. Int J Clin Pract Suppl. 2004;143:30-40. doi:10.1111/j.1368-504X.2004.00390.x

12. Ullah F, Afridi AK, Rahim F, Rahman SU, Ashfaq M. Efficacy of 5 $\mathrm{Mg}$ and $10 \mathrm{Mg}$ rosuvastatin in type-2 diabetes mellitus with hypercholesteroalemia. J Ayub Med Coll Abbottabad. 2015;27 (3):564-568.

13. Wolffenbuttel BH, Franken AA, Vincent HH, Dutch Corall Study G. Cholesterol-lowering effects of rosuvastatin compared with atorvastatin in patients with type 2 diabetes - CORALL study. J Intern Med. 2005;257(6):531-539. doi:10.1111/j.1365-2796.2005.01499.x

14. Olsson AG, McTaggart F, Raza A. Rosuvastatin: a highly effective new HMG-CoA reductase inhibitor. Cardiovasc Drug Rev. 2002;20 (4):303-328.

15. Choi HY, Lim HS, Kim YH, et al. Evaluation of the pharmacokinetics of the DPP-4 inhibitor gemigliptin when coadministered with rosuvastatin or irbesartan to healthy subjects. Curr Med Res Opin. 2015;31(2):229-241. doi:10.1185/03007995.2014.980886 
16. Lee E, Ryan S, Birmingham B, et al. Rosuvastatin pharmacokinetics and pharmacogenetics in white and Asian subjects residing in the same environment. Clin Pharmacol Ther. 2005;78(4):330-341. doi:10.1016/j.clpt.2005.06.013

17. Tzeng TB, Schneck DW, Birmingham BK, et al. Population pharmacokinetics of rosuvastatin: implications of renal impairment, race, and dyslipidaemia. Curr Med Res Opin. 2008;24(9):2575-2585. doi:10.1185/03007990802312807

18. AstraZeneca. Label of CRESTOR (ROSUVASTATIN CALCIUM) Tablets; 2016.

19. Food and Drug Administration, Center for Drug Evaluation and Research (CDER). Bioequivalence Studies with Pharmacokinetic Endpoints for Drugs Submitted under an ANDA. Draft Guidance; 2013 .
20. Shin D, Cho YM, Lee S, et al. Pharmacokinetic and pharmacodynamic interaction between gemigliptin and metformin in healthy subjects. Clin Drug Investig. 2014;34(6):383-393. doi:10.1007/ s40261-014-0184-3

21. LG Chem. Clinical Study Protocol (LG-GSCL003). version 3.2. 2016. Unpublished.

22. Ahren B. DPP-4 inhibitors. Best Pract Res Clin Endocrinol Metab. 2007;21(4):517-533. doi:10.1016/j.beem.2007.07.005

23. Olsson AG, Pears J, McKellar J, Mizan J, Raza A. Effect of rosuvastatin on low-density lipoprotein cholesterol in patients with hypercholesterolemia. Am J Cardiol. 2001;88(5):504-508. doi:10.1016/s0002-9149(01)01727-1

\section{Publish your work in this journal}

Drug Design, Development and Therapy is an international, peerreviewed open-access journal that spans the spectrum of drug design and development through to clinical applications. Clinical outcomes, patient safety, and programs for the development and effective, safe, and sustained use of medicines are a feature of the journal, which has also been accepted for indexing on PubMed Central. The manuscript management system is completely online and includes a very quick and fair peer-review system, which is all easy to use. Visit http://www. dovepress.com/testimonials.php to read real quotes from published authors. 